\title{
Disentangling the complex broad-band X-ray spectrum of IRAS 13197-1627 with NuSTAR, XMM-Newton and Suzaku
}

\author{
D. J. Walton, ${ }^{1 \star}$ M. Brightman, ${ }^{2}$ G. Risaliti, ${ }^{3}$ A. C. Fabian, ${ }^{1}$ F. Fürst, ${ }^{4}$ F. A. Harrison, ${ }^{2}$ \\ A. Lohfink, ${ }^{1}$ G. Matt, ${ }^{5}$ G. Miniutti, ${ }^{6}$ M. L. Parker ${ }^{1}$ and D. Stern ${ }^{7}$ \\ ${ }^{1}$ Institute of Astronomy, University of Cambridge, Madingley Road, Cambridge CB3 OHA, UK \\ ${ }^{2}$ Space Radiation Laboratory, California Institute of Technology, Pasadena, CA 91125, USA \\ ${ }^{3}$ Dipartimento di Fisica e Astronomia, Universita di Firenze, via G. Sansone 1, I-50019 Sesto Fiorentino, Firenze, Italy \\ ${ }^{4}$ European Space Astronomy Centre (ESAC), Science Operations Departement, E-28692 Villanueva de la Cañada, Madrid, Spain \\ ${ }^{5}$ Dipartimento di Matematica e Fisica, Universita degli Stu di Roma Tre, via della Vasca Navale 84, I-00146 Roma, Italy \\ ${ }^{6}$ Centro de Astrobiología (CSIC-INTA), Dep. de Astrofísica, ESAC campus, Camino Bajo del Castillo s/n, E-28692 Villanueva de la Cañada, Spain \\ ${ }^{7}$ Jet Propulsion Laboratory, California Institute of Technology, Pasadena, CA 91109, USA
}

Accepted 2017 October 9. Received 2017 October 9; in original form 2017 June 7

\begin{abstract}
We present results from a coordinated XMM-Newton + NuSTAR observation of the type 1.8 Seyfert galaxy IRAS 13197-1627. This is a highly complex source, with strong contributions from relativistic reflection from the inner accretion disc, neutral absorption and further reprocessing by more distant material, and ionized absorption from an outflow. We undertake a detailed spectral analysis combining the broad-band coverage provided by $X M M$ Newton $+N u S T A R$ with a multi-epoch approach incorporating archival observations performed by $X M M-N e w t o n$ and Suzaku. Our focus is on characterizing the reflection from the inner accretion disc, which previous works have suggested may dominate the AGN emission, and constraining the black hole spin. Using lamppost disc reflection models, we find that the results for the inner disc are largely insensitive to assumptions regarding the geometry of the distant reprocessor and the precise form of the illuminating X-ray continuum. However, these results do depend on the treatment of the iron abundance of the distant absorber/reprocessor. The multi-epoch data favour a scenario in which the AGN is chemically homogeneous, and we find that a rapidly rotating black hole is preferred, with $a^{*} \geq 0.7$, but a slowly rotating black hole is not strongly excluded. In addition to the results for the inner disc, we also find that both the neutral and ionized absorbers vary from epoch to epoch, implying that both have some degree of inhomogeneity in their structure.
\end{abstract}

Key words: black hole physics - galaxies: active-X-rays: individual: IRAS 13197-1627.

\section{INTRODUCTION}

Relativistically broadened iron emission is often seen from active galactic nuclei (AGN), both from individual sources (e.g. Tanaka et al. 1995; Fabian et al. 2009; Reis et al. 2014; Reynolds et al. 2014; $\mathrm{Xu}$ et al. 2017) and stacked spectra from AGN samples (e.g. Walton et al. 2015; Mantovani, Nandra \& Ponti 2016). Most AGN also show a 'hard' excess above $10 \mathrm{keV}$ consistent with Compton reflection from the accretion disc (e.g. Walton, Reis \& Fabian 2010; Nardini et al. 2011; Rivers, Markowitz \& Rothschild 2013). Together, these disc reflection features offer an opportunity to constrain the spins of the supermassive black holes that power these sources (e.g. Fabian et al. 1989; Laor 1991; Dauser et al. 2014), providing a rare observational window into their formation history (e.g. Volonteri et al. 2013;

^E-mail: dwalton@ @rl.caltech.edu
Dubois, Volonteri \& Silk 2014; Sesana et al. 2014). To date, spin estimates for $\sim 30$ AGN have been obtained through study of disc reflection, suggesting a preference for rapidly rotating black holes (e.g. Walton et al. 2013; Reynolds 2014; Vasudevan et al. 2016). However, the sample is relatively small and still not well defined in a statistical sense. Strong selection biases likely exist, as higher spin sources are expected to be brighter for a given rate of accretion (Brenneman et al. 2011; Reynolds et al. 2012), and it is also easier to obtain tight constraints on the spin parameter for more rapidly rotating black holes (Walton et al. 2013; Bonson \& Gallo 2016). It is therefore vital to further expand the sample of spin measurements such that these biases may be overcome.

In part, the sample size is hindered because the detection and characterization of emission from the inner disc can be complicated by both absorption from material along our line of sight to the inner accretion flow and additional reprocessing by more distant structures, which can also introduce strong spectral features 
Table 1. Details of the X-ray observations of IRAS 13197-1627 considered in this work.

\begin{tabular}{|c|c|c|c|c|c|}
\hline Epoch & Mission & OBSID & $\begin{array}{l}\text { Start } \\
\text { date }\end{array}$ & $\begin{array}{l}\text { Exposure }^{a} \\
\quad(\mathrm{ks})\end{array}$ & $\begin{array}{l}\text { Count rate } \\
\left(\text { count s }^{-1}\right)\end{array}$ \\
\hline \multirow[t]{2}{*}{ XN1 } & $\begin{array}{c}\text { NuSTAR } \\
\text { XMM-Newton }\end{array}$ & $\begin{array}{c}2016 \\
60101020002 \\
0763220201\end{array}$ & 2016-01-17 & $\begin{array}{c}85 \\
107 / 132\end{array}$ & $\begin{array}{l}0.121 \pm 0.001 \\
0.118 \pm 0.001\end{array}$ \\
\hline & & Archival & & & \\
\hline $\mathrm{X} 1$ & $X M M-N e w t o n$ & 0206580101 & 2005-01-24 & $32 / 39$ & $0.166 \pm 0.002$ \\
\hline $\mathrm{X} 2$ & $X M M-N e w t o n$ & 0506340101 & 2008-01-24 & $57 / 76$ & $0.179 \pm 0.002$ \\
\hline S1 & Suzaku & 704022010 & 2009-07-01 & $42 / 34$ & $0.045 \pm 0.001$ \\
\hline S2 & Suzaku & 707027010 & 2013-01-04 & $156 / 127$ & $0.0184 \pm 0.0004$ \\
\hline
\end{tabular}

$\overline{{ }^{a} X M M-N e w t o n}$ exposures are listed for the EPIC-pn/MOS detectors, and Suzaku exposures are given for the XIS/PIN detectors.

${ }^{b}$ Observed count rates are given in the 3-10 keV band for XMM-Newton (EPIC-pn) and Suzaku (XIS0), and the $3.5-79 \mathrm{keV}$ band for NuSTAR (FPMA).

in the X-ray band (e.g. Miller, Turner \& Reeves 2008, 2009; Sim et al. 2010). Robustly disentangling the absorption and reflection requires both sensitive coverage across all the key reflection features and good spectral resolution. As such, the combination of $X M M-N e w t o n$ and NUSTAR provides a particularly powerful tool for the study of AGN. The continuous 3-79 keV bandpass of NuSTAR (Harrison et al. 2013) is well suited to the study of reflection, while the better spectral resolution of XMM-Newton (Jansen et al. 2001) helps to separate out the broadened iron emission from the signatures of absorption. Even in the cases that exhibit complex absorption, as is seen for example in the well-studied Seyfert galaxy NGC 1365 (e.g. Rivers et al. 2015), coordinated XMM-Newton and NUSTAR observations can often isolate the relativistic reflection from the inner disc (e.g. Risaliti et al. 2013; Walton et al. 2014).

IRAS 13197-1627 (also known as MCG-03-34-64) is a source of particular interest in this respect. IRAS 13197-1627 is a bright, nearby $(z=0.01654)$, type 1.8 Seyfert AGN with a complex Xray spectrum. Previous observations with XMM-Newton, Suzaku, BeppoSAX and ASCA have shown that IRAS 13197-1627 is typically absorbed by a moderate neutral column $\left(N_{\mathrm{H}} \sim 5 \times 10^{23} \mathrm{~cm}^{-2}\right.$, although on rare occasions it can exhibit a much greater level of obscuration, reaching column densities up to $N_{\mathrm{H}} \sim 10^{24} \mathrm{~cm}^{-2}$ ), and that it also exhibits evidence for a relativistically broadened iron line from the innermost accretion flow (Dadina \& Cappi 2004; Miniutti et al. 2007; Brightman \& Nandra 2011). In addition to the broad iron line, there is evidence for narrow absorption features from blueshifted ionized iron at $\sim 7-7.5 \mathrm{keV}$, and a clear narrow core to the iron emission. In many respects, IRAS 13197-1627 therefore shows many similarities to the NGC 1365. Most importantly, the high-energy data from the non-imaging Suzaku PIN and BeppoSAX PDS detectors show evidence for an extremely strong excess over the expected power-law-like AGN continuum (e.g. Tatum et al. 2013), potentially suggesting an unusually strong contribution from reflection (Miniutti et al. 2007). If this is correct, the implied accretion geometry is rather extreme, requiring a compact $\mathrm{X}$-ray source located very close to the black hole such that gravitational lightbending can suppress the primary continuum emission relative to the reflection from the inner disc (e.g. Miniutti \& Fabian 2004). However, IRAS $13197-1627$ is located $\sim 2$ arcmin away from another active galaxy, MCG-3-34-63, potentially resulting in source confusion issues with the older non-imaging hard X-ray PIN and PDS detectors. Hard X-ray imaging spectrometers are required to unambiguously confirm that the flux observed by these detectors is associated with IRAS 13197-1627.
Here we present results from a coordinated broad-band observation of IRAS 13197-1627 with both NuSTAR and XMM-Newton in order to disentangle the complex X-ray spectrum exhibited by this source and test the extreme reflection scenario. The rest of the paper is structured as follows: in Section 2 we describe the $X M M$ Newton and NUSTAR observations and outline our data reduction procedure, in Sections 3 and 4 we present our analysis of these data, and in Section 5 we discuss the results obtained and summarize our conclusions.

\section{OBSERVATIONS AND DATA REDUCTION}

\subsection{Observation}

IRAS 13197-1627 was observed simultaneously with NuSTAR and XMM-Newton and on 2016 January 17; see Table 1 for details. The following sections detail our reduction of the data from this coordinated observation, referred to hereafter as epoch XN1.

\subsubsection{NUSTAR}

We reduced the NUSTAR data following standard procedures, first cleaning the data with NUPIPELINE, part of the NuSTAR Data Analysis Software (NUSTARDAS, v1.6.0). We used the standard depth correction, which significantly reduces the internal high-energy background, and also removed passages through the South Atlantic Anomaly. Instrumental calibration files from NuSTAR caldb v20160824 are used throughout this work. Source and background spectra/light curves and instrumental responses were then produced for each of the two focal plane modules (FPMA/B) using NUPRODUCTS. Source products were obtained from circular regions of radius 60 arcsec, and background was estimated from larger regions of blank sky on the same detector as IRAS 13197-1627. In order to maximize the signal-to-noise $(\mathrm{S} / \mathrm{N})$, we also extracted the 'spacecraft science' (mode 6) data in addition to the standard 'science' (mode 1) data, following the method outlined in Walton et al. (2016), which in this case provides $\sim 10$ percent of the total $\sim 85 \mathrm{ks}$ good NUSTAR exposure. IRAS $13197-1627$ is detected across the entire NuSTAR band (the S/N above $30 \mathrm{keV}$ is $\sim 20$ for each FPM), and we analyse the NuSTAR data between 3.5 and $79 \mathrm{keV}$ owing to a slight ( $\sim 40$ per cent) discrepancy between NUSTAR and XMM-Newton below $3.5 \mathrm{keV}$ in FPMB. This is likely related to the fact that the point spread function (PSF) is energy dependent at very low energies (Madsen et al. 2015), and on FPMB the PSF for IRAS 13197-1627 
straddles a chip gap, resulting in an uncertain PSF correction below $\sim 3.5 \mathrm{keV}$ in this case.

\subsubsection{XMM-Newton}

The XMM-Newton data reduction was carried out with the XMMNewton Science Analysis System (SAS v15.0.0), following the standard prescription provided in the online guide. ${ }^{1}$ Raw data files were cleaned using EPCHAIN for the EPIC-pn detector (Strüder et al. 2001), and EMCHAIN for two EPIC-MOS units (Turner et al. 2001). Source products were extracted from the cleaned event files from circular regions of radius 40 and 45 arcsec for EPIC-pn and EPIC-MOS, respectively, using XMMSELECT. As with the NuSTAR data, background was estimated from larger regions of blank sky on the same chip as IRAS 13197-1627. Only single and double events were considered for EPIC-pn and single to quadruple events were considered for EPIC-MOS. Periods of high background were excluded as standard. Instrumental response files for each of the detectors were generated with RMFGEN and ARFGEN. After performing the reduction separately for the two EPIC-MOS detectors, and confirming their consistency, these data were combined into a single EPIC-MOS spectrum using ADDASCASPEC.

\subsection{Archival data}

In addition to the new 2016 observation, we also analyse several archival observations of IRAS 13197-1627, focusing on high-S/N data obtained with the more recent generation of X-ray observatories. IRAS 13197-1627 was observed twice with XMM-Newton alone (epochs X1, X2), and also twice by Suzaku (epochs S1, S2; see Table 1). Epochs $X 1$ and S2 have previously been studied by Miniutti et al. (2007) and Brightman \& Nandra (2011), and by Tatum et al. (2013), respectively. The following sections provide details on our reduction of these data.

\subsubsection{XMM-Newton}

Our reduction procedure for the archival XMM-Newton observations largely follows that of the 2016 data, outlined in Section 2.1.2. For OBSID 0206580101 (epoch X1), we used the same source extraction regions as for the 2016 data. However, for OBSID 0506340101 (epoch X2), we used slightly smaller source regions of radius 30 arcsec for the EPIC-pn detector to avoid chip gaps, as IRAS 13197-1627 was placed slightly off-axis.

\subsubsection{Suzaku}

The data from the two Suzaku observations were reduced using the HEASOFT software package (v6.19), following the procedure outlined in the Suzaku Data Reduction Guide. ${ }^{2}$ To extract science products from the XIS units (Koyama et al. 2007), we reprocessed the unfiltered event files for each of the operational CCDs (XIS0, 1, 3) and editing modes $(3 \times 3,5 \times 5)$. Cleaned event files were generated by running the $S u z a k u$ pipeline with the latest calibration and screening criteria files (XIS caldb v20160607). Source products were extracted from circular regions $\sim 150$ arcsec in radius using XSELECT, and the background was extracted from adjacent regions free of any contaminating sources, with care taken to avoid the calibration sources in the corners. Instrumental response files were generated for each detector using the XISRESP script with a medium resolution. The spectra and response files for the two front-illuminated (FI) detectors (XIS0, 3) were combined using the FTOOL ADDASCASPEC; the XIS1 unit is the only back-illuminated detector.

For the HXD PIN detector (Takahashi et al. 2007), we also reprocessed the unfiltered event files with the latest calibration/screening files (HXD caldb v20110913). The HXD is a collimating instrument, so background estimation requires separate consideration of both the non-X-ray instrumental background (NXB) and the cosmic X-ray background (CXB). The instrumental response and NXB background files are provided by the Suzaku team for each observation; ${ }^{3}$ in this work, we use the higher quality 'tuned' (Model D) background model. The final spectral products were generated using the HXDPINXBPI script which, in addition to extracting the source spectrum, adds a simulated contribution from the CXB to the NXB (using the form of Boldt 1987) to produce a total background spectrum. We analyse the PIN data over the $\sim 15-50 \mathrm{keV}$ bandpass.

\section{NUSTAR IMAGING}

As noted previously, IRAS $13197-1627$ is located $\sim 2$ arcmin away from MCG-3-34-63, another known X-ray emitting active galaxy $(z=0.02133$, classified as a Seyfert 2). While the latter AGN is significantly fainter than IRAS 13197-1627 in the soft X-ray band (typically by more than a factor of 100), sources with extremely hard spectra that are only really seen in hard X-rays are known (e.g. Lansbury et al. 2017), and the only previous hard X-ray ( $>10 \mathrm{keV}$ ) observations of this field have been with non-imaging instruments (the PDS detector on BeppoSAX and the PIN detector on board Suzaku; Miniutti et al. 2007; Tatum et al. 2013), leaving a major uncertainty over their relative contributions in the band in which the hard excess is seen. NUSTAR is the first hard X-ray mission with sufficient imaging capabilities to resolve IRAS 13197-1627 and MCG-3-34-63. We show the NuSTAR image in Fig. 1. It is clear that the hard X-ray flux is entirely dominated by IRAS 13197-1627 (MCG-3-34-63 is not even detected), confirming that the hard Xray emission recorded by BeppoSAX and Suzaku is indeed associated with IRAS 13197-1627. Assuming that IRAS 13197-1627 constantly outshines MCG-3-34-63 by a significant factor at these energies, we adopt the standard cross-calibration constants of 1.18 for epoch S1 (performed with an XIS nominal pointing) and 1.16 for epoch S2 (performed with an HXD nominal pointing) for the Suzaku PIN detector with respect to the XIS units during our analysis of the archival data (Section 4.2).

\section{SPECTRAL ANALYSIS}

The majority of our work focuses on spectral analysis of the observations considered. Model fits are performed with XSPEC v12.6.0f (Arnaud 1996), and we quote parameter uncertainties at the 90 per cent confidence level for one interesting parameter. All models include a Galactic absorption component with a fixed column of $N_{\text {H,Gal }}=4.99 \times 10^{20} \mathrm{~cm}^{-2}$ (Kalberla et al. 2005), modelled with the TBNEW neutral absorption code (Wilms, Allen \& McCray 2000). We use the cross-sections of Verner et al. (1996) for the absorption, as recommended, but we combine these with the solar abundance set of Grevesse \& Sauval (1998) for internal self-consistency with both the XILLVER family of reflection models (García \& Kallman 2010)

\footnotetext{
${ }^{3}$ http://www.astro.isas.ac.jp/suzaku/analysis/hxd/
}

\footnotetext{
${ }^{1}$ https://www.cosmos.esa.int/web/xmm-newton

$2 \mathrm{http} / / /$ heasarc.gsfc.nasa.gov/docs/suzaku/analysis/
} 

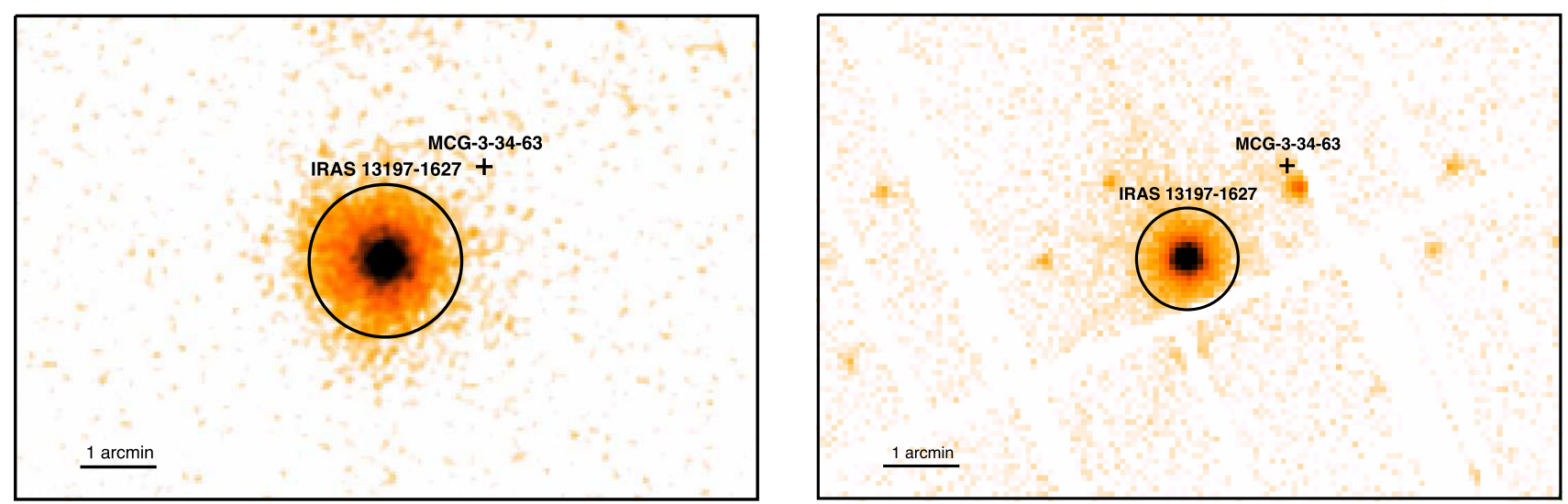

Figure 1. NuSTAR (left, FPMA, 3-79 keV) and XMM-Newton (right, EPIC-pn, 0.3-10 keV) images of IRAS 13197-1627. The position of the nearby AGN MCG-3-34-63, which is not detected by NuSTAR, is also shown. The hard X-ray flux from this field is dominated by IRAS 13197-1627. The circular regions indicate the source extraction regions used in each case.

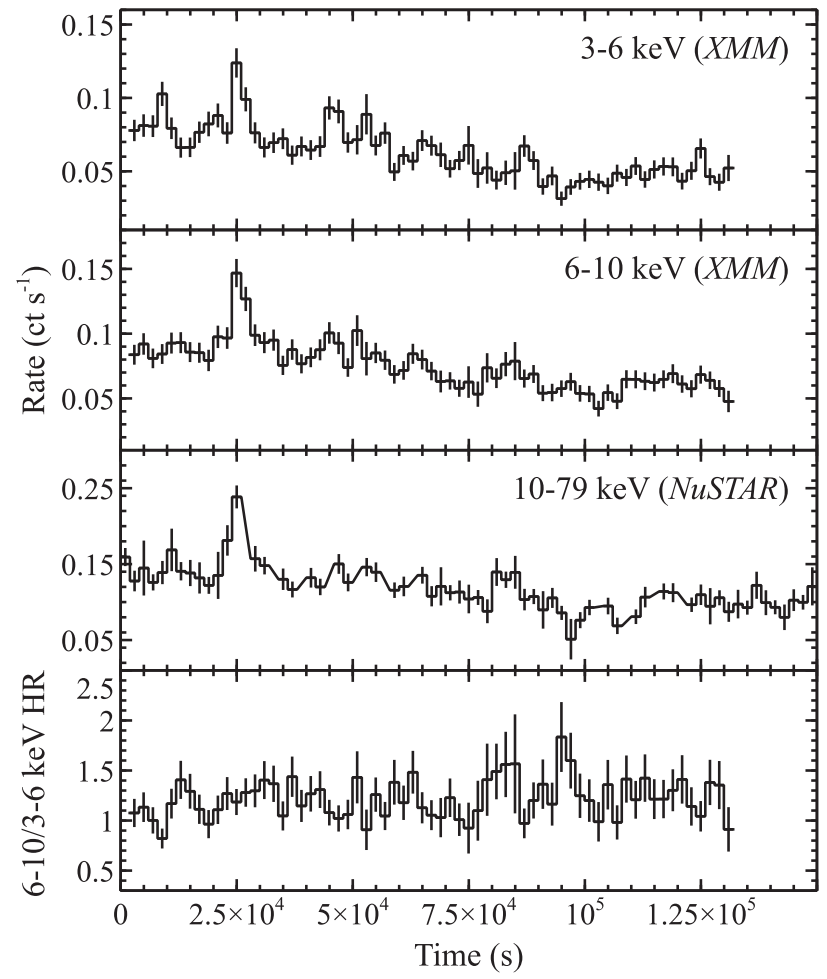

Figure 2. The light curves observed with the XMM-Newton EPIC-pn detector in the 3-6 and 6-10 keV bands, and with the NUSTAR FPMA detector in the $10-79 \mathrm{keV}$ band ( $2 \mathrm{ks}$ time bins; first three panels). The bottom panel shows the hardness ratio computed between the 6-10 and 3-6 keV XMMNewton bands. Although some flux variability is observed, including a small flare towards the beginning of the observation, no evidence for strong spectral variability is seen.

and the XSTAR photoionization code (Kallman \& Bautista 2001), which are heavily utilized throughout this work.

\subsection{Data}

We begin our analysis by focusing on the new coordinated observation taken with XMM-Newton $+N u S T A R(X N 1)$. In Fig. 2, we show $X M M-N e w t o n$ (EPIC-pn) and NuSTAR (FPMA) light curves from this observation extracted in the 3-6, 6-10 and 10-79 keV bands, as well as a simple hardness ratio computed as the ratio between the EPIC-pn count rates in the 6-10 and 3-6 keV bands. While there is some flux variability observed, including a short and fairly sharp flare towards the beginning of the observation that seen by both missions, there is little evidence for strong spectral variability, so we focus on modelling the time-averaged spectrum from this epoch. In our analysis of these data, cross-calibration uncertainties between the different detectors are accounted for by allowing multiplicative constants to float between the data sets, fixing EPIC-pn at unity. These constants are within $\sim 10$ per cent of unity, as expected (Madsen et al. 2015).

We show the full broad-band spectrum observed in the left-hand panel of Fig. 3. IRAS $13197-1627$ is known to be quite heavily absorbed, and during this epoch the hard AGN component only dominates above $3 \mathrm{keV}$; at lower energies the observed emission is dominated by a combination of photoionized gas and thermal plasma emission (Miniutti et al. 2007). Since we are interested in the emission from the AGN in this work, we limit our analysis to energies above $3 \mathrm{keV}$. The right-hand panel of Fig. 3 shows the data/model ratio of the combined XMM-Newton+NuSTAR data set to a fairly typical $\Gamma=2$ AGN continuum, modified by a simple, partially covering neutral absorber (modelled with TBNEW_PCF, a partially covering version of the TBNEW absorption code), fitted to the $3-4,8-10$ and $50-78 \mathrm{keV}$ bands where the primary AGN continuum would be expected to dominate. The column density and covering factor are $\sim 7 \times 10^{23} \mathrm{~cm}^{-2}$ and $\sim 0.94$, respectively; adopting a different continuum slope pivots the residuals (and changes the absorption parameters) but does not change their overall shape. A strong, broad feature is clearly seen in the iron bandpass, and a strong excess of emission is observed above $10 \mathrm{keV}$, similar to the broad iron $\mathrm{K}$ emission and strong hard excess previously reported for this source (Dadina \& Cappi 2004; Miniutti et al. 2007; Tatum et al. 2013). In addition to these broad features, a narrow core to the iron emission at $6.4 \mathrm{keV}$ and a narrow absorption feature just above $7 \mathrm{keV}$ are both evident. IRAS $13197-1627$ is also known to exhibit absorption from ionized iron (Miniutti et al. 2007), and there is evidence for a second narrow absorption line at $\sim 6.7 \mathrm{keV}$ (this is not so visually obvious, but is also required by the data; see below); given the observed energies and the known redshift of IRAS 13197-1627 $(z=0.0165)$, these absorption lines are likely associated with Fe XXv and Fe XXVI from an outflowing disc wind. Prior studies have indicated an outflow velocity of $v_{\text {out }} \sim 5000 \mathrm{~km} \mathrm{~s}^{-1}$ (Miniutti et al. 2007). 

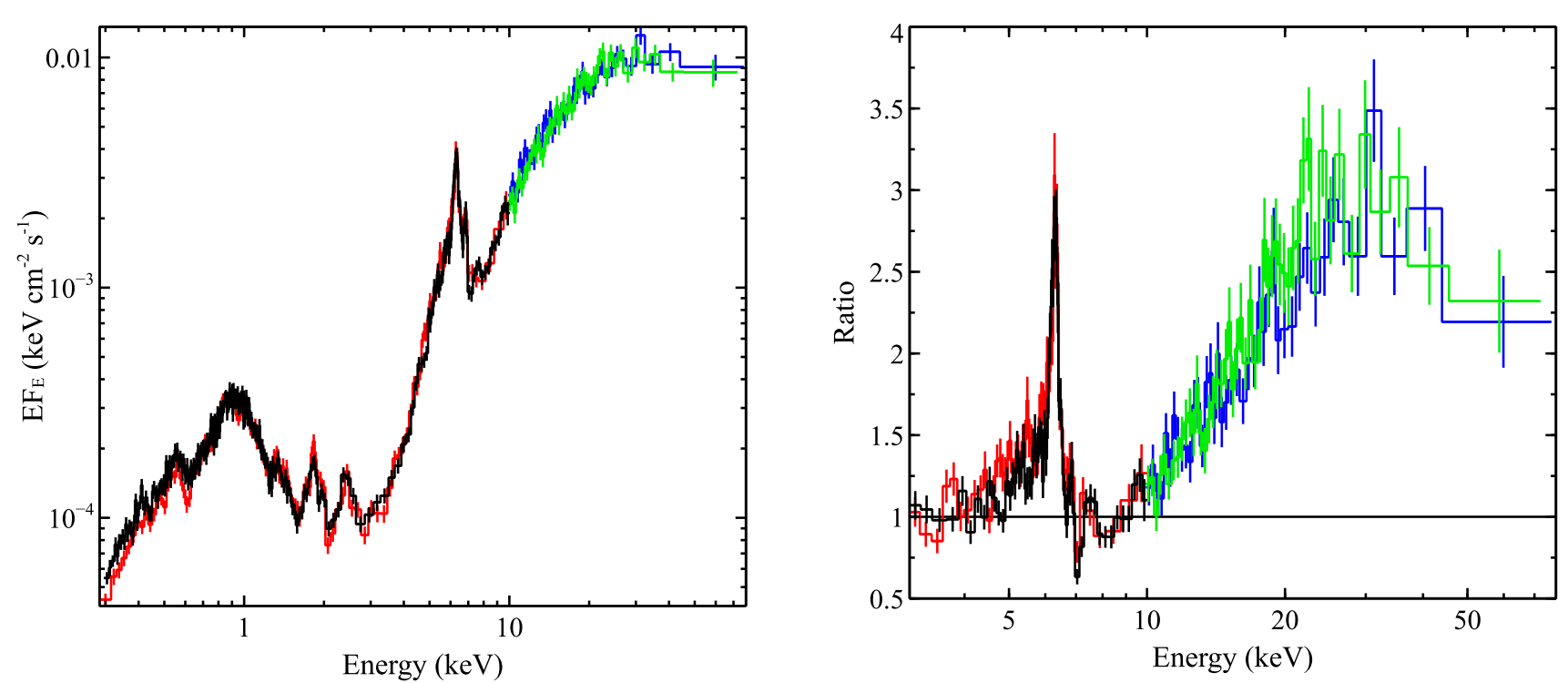

Figure 3. Left-hand panel: time-averaged spectra from our coordinated NuSTAR+XMM-Newton observation of IRAS 13197-1627, unfolded through a model that is constant with energy. Data from the EPIC-pn and EPIC-MOS detectors on XMM-Newton and the FPMA and FPMB modules on NuSTAR are shown in black, red, green and blue, respectively, and for clarity we show the XMM-Newton data below $10 \mathrm{keV}$, and the NuSTAR data above $10 \mathrm{keV}$. At low energies, the emission is dominated by photoionized plasmas rather than the intrinsic AGN emission, so we focus our analysis above $3 \mathrm{keV}$. Right-hand panel: residuals to a simple $\Gamma=2.0$ power-law continuum, modified by partially covering neutral absorption, and applied to the $3-4,8-10$ and $50-78 \mathrm{keV}$ energy ranges. The hallmarks of relativistic reflection from the inner accretion disc, i.e. a broadened iron line at $\sim 6 \mathrm{keV}$ and a strong Compton hump at $\sim 30 \mathrm{keV}$, are seen. The data in both panels have been rebinned for visual purposes.

In order to fit the AGN spectrum, we therefore construct models consisting of a partially covering neutral absorber, a powerlaw/Compton-scattered continuum, reflection from the accretion disc, reflection from more distant material and an ionized absorber. We assume that the distant reflection arises from the same structure that results in the neutral absorption, which is likely the putative torus invoked in AGN unification models. We therefore group the model components into 'distant' components (neutral absorption, distant reflection) and 'inner' components (primary continuum, disc reflection, ionized absorption), and set the models up such that the neutral absorption acts on all of the inner components, but does not act on the distant reflection.

\subsubsection{Model 1: basic approach}

In this work, we model the innermost regions of the AGN with a simple lamppost geometry using the RELXILL family of disc reflection models (García et al. 2014), which merges the XILLVER family of reflection models with the RELCONV model for the relativistic effects relevant for regions close to a black hole (Dauser et al. 2010). The lamppost geometry treats the X-ray emitting region as a point source situated on the rotation axis of the black hole. While this is obviously an idealized geometric approximation, it provides a simple parametrization for the reflected emission and allows us to exclude low-spin, reflection-dominated solutions that are unphysical for thin-disc accretion (Dauser et al. 2014). We start by using the RELXILLLP model, which treats the illuminating continuum as a simple power law with a high-energy exponential cut-off. This model incorporates both the primary continuum and the reflected emission from the accretion disc, and self-consistently determines both the radial emissivity profile for the disc and the relative strength of the reflected emission ( $R_{\text {frac }}$, defined to be the ratio between the continuum fluxes seen by the disc and by the observer in the latest versions of the RELXILLLP model; see Dauser et al. 2016) based on the spin of the black hole ( $a^{*}$; we assume that the disc extends into the innermost stable circular orbit) and the height of the X-ray source ( $h$; see also Wilkins \& Fabian 2012). In order to ensure that the source is always required to be outside the event horizon, we fit the model with $h$ in units of the vertical event horizon radius $\left(R_{\mathrm{H}}\right.$, which varies from $1-2 R_{\mathrm{G}}$ for maximally rotating to non-rotating black holes, where $R_{\mathrm{G}}=G M / c^{2}$ is the gravitational radius). The other key free parameters are the photon index and high-energy cut-off of the illuminating continuum $\left(\Gamma, E_{\mathrm{cut}}\right)$, the iron abundance $\left(A_{\mathrm{Fe}}\right)$, inclination (i) and ionization parameter $(\xi)$ of the accretion disc. Here, the ionization parameter is defined as $\xi=4 \pi F_{X} / n$, where $F_{X}$ is the incident X-ray flux and $n$ is the density of the material.

The neutral absorption is again modelled with TBNEW_PCF, and we initially treat the distant reflection with an unblurred XILLVER component. TBNEW_PCF implicitly assumes that the absorber has solar abundances, and only has the column density $\left(N_{\mathrm{H}}\right)$ and the covering fraction $\left(C_{\mathrm{f}}\right)$ as free parameters (we assume that the neutral absorber is at the redshift of the host galaxy). The XILLVER model assumes a simple slab geometry for the reflector, and its key free parameters are again the photon index and high-energy cut-off of the illuminating continuum, the ionization parameter and the iron abundance of the reflecting medium, and the inclination of the slab. Given the assumed association between the distant reflector and the neutral absorber, we fix the iron abundance for the XILLVER component to solar. The parameters for the illuminating continuum are assumed to be the same as those for the RELXILLLP component, and we fix the inclination to $45^{\circ}$, since the fits are largely insensitive to this parameter. In order to provide the reader with an indication of the relative contribution of the distant reflection, in addition to their normalizations we also present the ratio of the observed fluxes for the distant reflector and the RELXILLLP component $\left(F_{\text {dist/relx }}\right)$ in the $20-40 \mathrm{keV}$ band, roughly where the Compton hump peaks; the effects of photoelectric absorption from the neutral absorber are also reduced in this band. In addition to treating the photoelectric 
Table 2. Results obtained for the free parameters in the various lamppost reflection models fitted to epoch XN1.

\begin{tabular}{|c|c|c|c|c|c|c|}
\hline \multirow[t]{2}{*}{ Model component } & \multicolumn{2}{|c|}{ Parameter } & \multicolumn{4}{|c|}{ Model } \\
\hline & & & 1 & 2 & 3 & 4 \\
\hline \multirow[t]{2}{*}{ TBNEW_PCF } & $N_{\mathrm{H}}$ & {$\left[10^{23} \mathrm{~cm}^{-2}\right]$} & $8.1 \pm 0.4$ & $7.6 \pm 0.4$ & $6.2_{-0.5}^{+0.3}$ & $6.4_{-0.4}^{+0.2}$ \\
\hline & $A_{\mathrm{Fe}, \text { distant }}$ & [solar] & $=1$ & $=1$ & $=A_{\mathrm{Fe}, \mathrm{disc}}$ & $=A_{\mathrm{Fe}, \mathrm{disc}}$ \\
\hline \multirow[t]{7}{*}{ RELXILLLP (CP) } & $\Gamma$ & & $2.16_{-0.37}^{+0.10}$ & $2.1_{-0.2}^{+0.3}$ & $2.2_{-0.2}^{+0.1}$ & $2.12_{-0.32}^{+0.04}$ \\
\hline & $E_{\text {cut }}$ or $k T_{\mathrm{e}}^{a}$ & {$[\mathrm{keV}]$} & $150_{-40}^{+100}$ & $100_{-40}^{+300}$ & $>110$ & $>48$ \\
\hline & $i$ & {$\left[{ }^{\circ}\right]$} & $44_{-3}^{+2}$ & $44_{-3}^{+2}$ & $61_{-3}^{+2}$ & $60_{-3}^{+2}$ \\
\hline & $h$ & {$\left[R_{\mathrm{H}}\right]$} & $<1.4$ & $<1.4$ & $<1.8$ & $<1.8$ \\
\hline & $R_{\mathrm{frac}}^{c}$ & & $5.1_{-1.9}^{+3.3}$ & $3.0_{-0.4}^{+0.8}$ & $1.7_{-0.6}^{+1.7}$ & $1.6_{-0.7}^{+1.4}$ \\
\hline & $\log \xi$ & $\log \left[\mathrm{erg} \mathrm{cm} \mathrm{s}^{-1}\right]$ & $<1.3$ & $<1.5$ & $<2.3$ & $<3.0$ \\
\hline & $A_{\mathrm{Fe}, \mathrm{disc}^{b}}{ }^{2}$ & [solar] & $>7.1$ & $>7.3$ & $2.3_{-0.1}^{+0.2}$ & $2.3_{-0.1}^{+0.2}$ \\
\hline XSTARabs & $v_{\text {out }}$ & {$\left[\mathrm{km} \mathrm{s}^{-1}\right]$} & $4900_{-700}^{+600}$ & $4800 \pm 700$ & $4700 \pm 700$ & $4700 \pm 700$ \\
\hline \multirow[t]{2}{*}{ XILLVER (_CP) } & Norm & {$\left[10^{-5}\right]$} & $5.1_{-0.3}^{+0.8}$ & - & - & $3.3_{-1.2}^{+0.4}$ \\
\hline & $F_{\text {dist } / \text { relx }}{ }^{d}$ & & $0.19_{-0.02}^{+0.03}$ & - & - & $0.14 \pm 0.02$ \\
\hline \multirow[t]{3}{*}{ MYTORUS } & $N_{\mathrm{H}}$ & {$\left[10^{23} \mathrm{~cm}^{-2}\right]$} & - & $22_{-8}^{+16}$ & $>32$ & - \\
\hline & Norm & {$\left[10^{-2}\right]$} & - & $1.5_{-0.4}^{+0.7}$ & $0.9_{-0.2}^{+0.5}$ & - \\
\hline & $F_{\text {dist } / \text { relx }}^{d}$ & & - & $0.49_{-0.37}^{+0.20}$ & $0.19_{-0.04}^{+0.03}$ & - \\
\hline$\chi^{2} / \mathrm{DoF}$ & & & $794 / 723$ & $796 / 722$ & $789 / 722$ & $787 / 723$ \\
\hline
\end{tabular}

${ }^{a} E_{\text {cut }}$ is limited to $\leq 1000 \mathrm{keV}$ following García et al. (2015), and $k T_{\mathrm{e}}$ is limited by the bounds of the RELXILL_CP grid to $20 \leq k T_{\mathrm{e}} \leq 400 \mathrm{keV}$.

${ }^{b}$ The iron abundance is limited by the bounds of the XSTAR grid used here to $A_{\mathrm{Fe}} \leq 10.0$.

${ }^{c} R_{\text {frac }}$ is calculated self-consistently for a simple lamppost geometry from $a^{*}$ and $h$. Errors represent the range of values permitted by varying these parameters within their 90 per cent uncertainties.

${ }^{d}$ Ratio of the observed fluxes from the distant reflector and the RELXILLLP (_CP) components in the 20-40 keV band.

absorption from the neutral absorber, as the column along our line of sight to IRAS 13197-1627 can be quite large (see Section 4.2) we also account for flux losses due to Compton scattering in the intervening material by including a CABS component with the column set to be equal to that of the neutral absorber.

Finally, we model the ionized absorption with the XSTAR photoionization code, computing a custom grid of absorption models. We allow the column density, the ionization parameter, the outflow velocity and the iron abundance of the absorber to be varied as free parameters. The other abundances are assumed to be solar, and the absorbing medium is assumed to be illuminated by a $\Gamma=2$ power law for the model calculation. Initial modelling of the two absorption lines with Gaussian features (both lines are significant; the fit without any absorption features included is improved by $\Delta \chi^{2}=111$ for three additional free parameters when the Fe xxvI line is added, and by a further $\Delta \chi^{2}=27$ for one more free parameter when the Fe xxv line is added, assuming the same outflow velocity and broadening for the two lines) suggested line broadening of $\sigma \sim 0.06 \mathrm{keV}$, so we set the turbulent velocity to $1750 \mathrm{~km} \mathrm{~s}^{-1}$. Since we assume that the outflow comes from the accretion disc, we link the iron abundance of the ionized absorption to that of the disc reflection (RELXILLLP) component in our analysis. The final form of this initial model, which we refer to as Model 1 , is TBNEW $\times$ (XILLVER + $($ TBNEW_PCF $\times$ XSTAR $\times$ CABS $\times$ RELXILLLP)).

Applying this model to the combined $3-79 \mathrm{keV}$ spectrum from $\mathrm{XN1}$ results in a good fit, with $\chi^{2}=794$ for 723 degrees of freedom
(DoF). The parameter values obtained are given in Table 2. The neutral absorption column local to IRAS $13197-1627$ is found to be rather large $\left(N_{\mathrm{H}}=[8.1 \pm 0.4] \times 10^{23} \mathrm{~cm}^{-2}\right)$, but the fits still imply a very strong disc reflection contribution $\left(R_{\mathrm{frac}} \sim 5\right.$; for reference, a thin accretion disc in Newtonian gravity should give $R_{\text {frac }} \sim 1$ ), broadly similar to that inferred previously (Miniutti et al. 2007). For a standard thin accretion disc, strong gravitational lightbending is required to produce such strong reflection (e.g. Miniutti \& Fabian 2004). This in turn requires a very compact X-ray source in close vicinity to a rapidly rotating black hole, such that the majority of the emission from the X-ray source is bent on to the disc, rather than escaping to the observer directly (see also Dauser et al. 2014; Parker et al. 2014). The confidence contour for the black hole spin is shown in Fig. 4; we find $a^{*}=0.97_{-0.04}^{+0.01}$ for this model. We also find that, as expected, the height of the $\mathrm{X}$-ray source is constrained to be very small: $h \leq 1.6 R_{\mathrm{G}}$ (converting from $R_{\mathrm{H}}$ based on the spin constraints).

\subsubsection{Model 2: MYtorus}

The XILLVER model used to account for the distant reflection contribution in Model 1 assumes a simple slab geometry for the reprocessor, which is also treated as being highly optically thick. While convenient, this is unlikely to be physically realistic for the distant reprocessor in an AGN, which is expected to have a torus-like geometry and a finite column of material. Different geometries can lead 


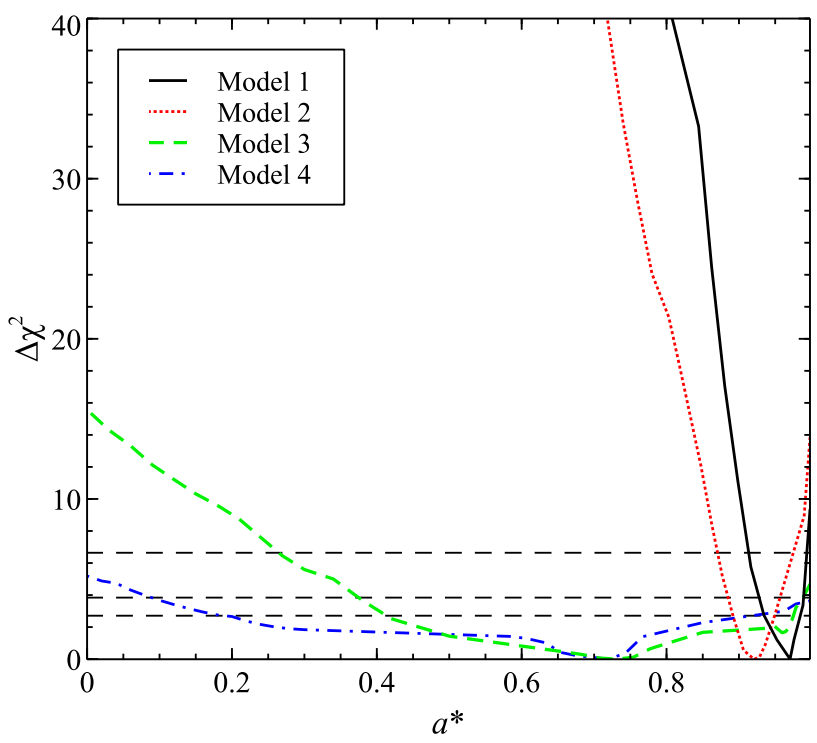

Figure 4. The $\Delta \chi^{2}$ confidence contours for the black hole spin obtained for Models 1-4 applied to epoch XN1. The horizontal dashed lines represent the 90,95 and 99 per cent confidence levels for a single parameter of interest.

to differences in the reflected spectra (e.g. Brightman et al. 2015). In order to determine whether this could have any influence on the results obtained for the disc reflection, we construct a second model (hereafter Model 2) in which the XILLVER component is replaced by MYTORUs (Murphy \& Yaqoob 2009). This self-consistently computes the absorption and reprocessed emission from a neutral torus surrounding a central illuminating X-ray source. MYTORUs assumes a doughnut-like geometry such that the line-of-sight column density varies with viewing angle, up to a maximum equatorial column density. The basic version of MYTORUs also assumes the illuminating continuum to be a power law (up to a termination energy of $500 \mathrm{keV}$, which is outside the NuSTAR bandpass), that the torus has a solar composition (based on the solar abundances reported in Anders \& Grevesse 1989) and that the torus has an opening of $30^{\circ}$ from the equatorial plane. The key MYTORUs parameters are the equatorial column density, the angle at which the torus is viewed and the continuum photon index.

The $30^{\circ}$ opening angle assumed for the torus is equivalent to a covering factor of $\Omega=2 \pi$, or in fractional terms $\Omega_{\mathrm{f}}=0.5$ (where $\Omega_{\mathrm{f}}=\Omega / 4 \pi$ ). Although this covering factor is fixed in the available MYTORUs models, the assumed value is quite reasonable for IRAS 13197-1627. Correcting for losses from both photoelectric absorption and Compton scattering due to the neutral line-ofsight absorber, we estimate the intrinsic $2-10 \mathrm{keV}$ luminosity to be $\sim 1.5 \times 10^{43} \mathrm{erg} \mathrm{s}^{-1}$ during epoch XN1. Based on the correlation between the covering factor of the distant reprocessor and the intrinsic $2-10 \mathrm{keV}$ X-ray luminosity presented by Brightman et al. (2015), we find an expected covering factor of $\Omega_{\mathrm{f}} \sim 0.5$.

The MYTORUs model is separated into three different components: the absorption from the torus, the continuum component to the reprocessed emission and the reprocessed line emission. The line component only includes Fe $\mathrm{K} \alpha$ and $\mathrm{K} \beta$, but these are the key transitions relevant to this work. In order to continue allowing for the possibility of partial covering, we still model the neutral absorption with TBNEW_PCF, but we replace the XILLVER component with a combination of the two reprocessed MYTORUs components (lines plus continuum). All parameters are required to be the same for both, but we additionally multiply the line emission with an energy- independent constant factor of 0.68 [the ratio of the Anders \& Grevesse (1989) and Grevesse \& Sauval (1998) solar iron abundances] to approximately account for the differences in the abundance sets used for MYTORUS and XILLVER/XSTAR, since the iron abundance is not currently a free parameter in MYTORUs. ${ }^{4}$ Since we are only using the reprocessed MYTORUS components, the fits are not strongly sensitive to the viewing angle for the torus. However, as the neutral column varies from epoch to epoch (see below), we assume that we are viewing the system fairly close to the edge of the torus, and set this to $65^{\circ}$. Finally, we link the photon index to that of the RELXILLLP component.

This model provides a similarly good fit to the data as Model 1 (see Table 2). Critically, although there are some minor quantitative changes to some of the parameters, the results are generally similar to Model 1. The reflection fraction is still high $\left(R_{\text {frac }} \sim 3\right)$, requiring a strong degree of lightbending, and again a compact $\mathrm{X}$-ray source and a rapidly rotating black hole. In this case, we find $a^{*}=0.92 \pm 0.03$ (see Fig. 4). The key results for the disc reflection do not depend strongly on the precise modelling of the distant reflection. The best-fitting MYTORUS column density suggests that while the neutral absorption along our line of sight is Compton thin, the absorbing medium does become Compton thick closer to the equatorial plane.

\subsubsection{Model 3: common iron abundance}

One issue of note with both Models 1 and 2 is that the iron abundance of the disc is found to be very high. Similar iron abundances have been found in other AGN, e.g. 1H 0707-495 (Fabian et al. 2009) and IRAS 13224-3809 (Fabian et al. 2013). However, in this case, the iron abundance of the disc strongly contrasts the solar abundance assumed for the distant model components (neutral absorption, distant reflection) which play a significant role in shaping the observed spectrum (in contrast to IRAS 13197-1627, both 1H0707-495 and IRAS 13224-3809 are unobscured systems). This is similar to results recently reported by Xu et al. (2017) for the Seyfert 2 galaxy IRAS 05189-2524.

In order to investigate this issue further, we construct a third model in which all the distant and the disc components have a common iron abundance (hereafter Model 3), mimicking the approach taken in Miniutti et al. (2007). We continue to model the distant reflection with MYTORUS here, but stress that again similar results are seen if we replace this with XILLVER. Since the TBNEW_PCF model does not include iron abundance as a free parameter, in order to account for the neutral absorption we replace this with a version of TBNEW in which the iron abundance can be varied, convolved with a PARTCOV component to continue allowing for the possibility of partial covering (for simplicity, we still refer to this combination as TBNEW_PCF in Table 2). We then link all the iron abundance parameters together, along with the constant multiplicative factor applied to the MYTORUs line emission (continuing to include the additional factor of 0.68 to account for the different solar iron abundance assumed by MYTORUs).

This model provides a similarly good fit to the data as both Models 1 and 2 (again, see Table 2). However, there are some significant differences of note between the results obtained. The iron abundance is still super-solar $\left(A_{\mathrm{Fe}}=2.3_{-0.1}^{+0.2}\right)$, but not extremely so, and is (unsurprisingly) intermediate to the solar abundance assumed for

\footnotetext{
${ }^{4}$ Multiplying the line component by a constant is only a rough approximation for a different iron abundance as the strength of the iron absorption edge should also vary correspondingly; see the MYTORUS manual for caveats.
} 

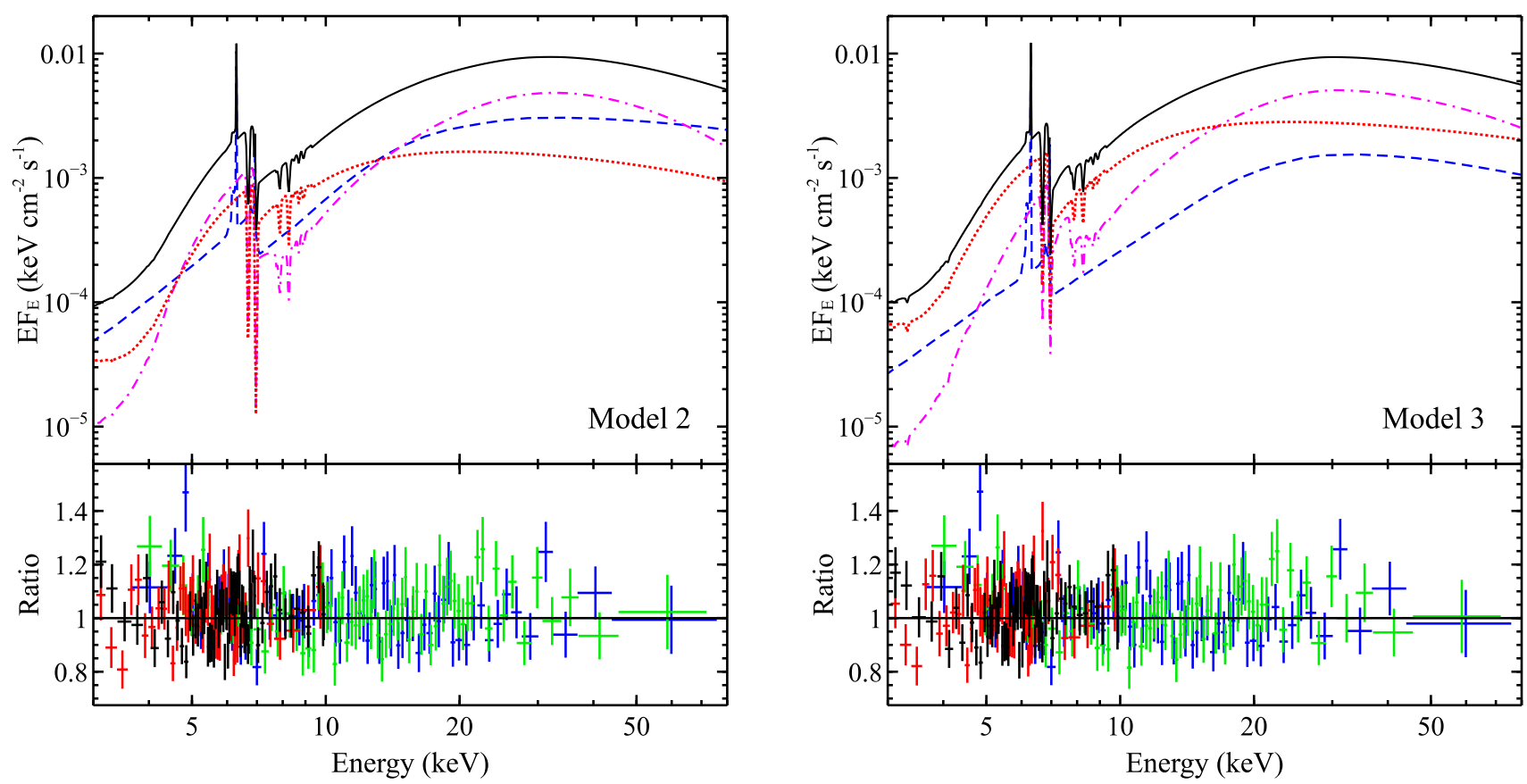

Figure 5. A comparison of the best-fitting models for epoch XN1 assuming a torus geometry for the distant reprocessor. Left-hand panels: a solar iron abundance assumed for the distant model components. Right-hand panels: assuming a common iron abundance for all AGN model components. Top panels: the relative contributions of the different model components, with the total model shown in solid black, the power-law continuum in dotted red, the inner disc reflection in dash-dotted magenta and distant reflection in dashed blue. Bottom panels: data/model ratios for these models. The colour scheme for these panels is the same as Fig. 3, and again the data have been rebinned for visual purposes.

the distant components and the high abundance found for the disc components in the previous models. This allows the neutral absorption to account for more of the strong drop in the spectrum above $\sim 7 \mathrm{keV}$ (the iron edge) and produce more curvature in the observed continuum above $10 \mathrm{keV}$, which reduces the requirement for the reflection model to account for these features with a large reflection fraction. In turn, this means the requirement for a rapidly rotating black hole is no longer as strong, and so the parameter constraints are correspondingly looser. In this case, we find that $a^{*}=0.73_{-0.32}^{+0.25}$ (see Fig. 4). However, the best-fitting reflection fraction is still greater than unity, so the X-ray source is still required to be relatively compact. The increased iron abundance in the absorber also means that the blue wing of the iron line in the disc reflection component is no longer required to be at a low enough energy to help produce the $\sim 7 \mathrm{keV}$ spectral drop, which results in a higher bestfitting inclination. Lastly, the stronger line emission relative to the continuum in the distant reflection means this component cannot account for as much of the observed emission at $\sim 3-4 \mathrm{keV}$ as in the previous models, and so the column of the neutral absorber is now significantly lower to compensate. A comparison of Models 2 and 3 is shown in Fig. 5.

\subsubsection{Model 4: compton-scattered continuum}

All three of the models presented so far have treated the primary continuum as a simple power law with an exponential high-energy cut-off. The reflection components used so far have also been calculated assuming the illuminating continuum to be a simple power-law continuum either with (RELXILLLP, XILLVER) or without (MYTORUS) an exponential high-energy cut-off. However, the nature of the primary $\mathrm{X}$-ray continuum is widely expected to be Compton upscattering of low-energy photons from the accretion disc by hot electrons for most AGN (e.g. Haardt \& Maraschi 1991). While a cut-off power law is often a reasonable approximation for a real Comptonscattered continuum, there are subtle differences between them. The former is constantly curving across all energies, while the latter is more power-law-like until it rolls over with a sharper cut-off (e.g. Zdziarski et al. 2003; Fabian et al. 2015; Fürst et al. 2016).

Recently, versions of the XILLVER family of models have been calculated using a realistic Compton-scattered continuum for the illuminating continuum (hereafter XILLVER_CP, etc.). Therefore, we construct one more model utilizing these new versions to investigate what effect the differences between the assumed continuum forms might have on the results for the disc reflection (Model 4), replacing RELXILLLP with RELXILLLP_CP, and since the results with MYTORUS and XILLVER have been consistent, replacing MYTORUS with XILLVER_CP for convenience. ${ }^{5}$ XILLVER_CP and RELXILLLP_CP have been calculated using the NTHCOMP thermal Comptonization model (Zdziarski, Johnson \& Magdziarz 1996; Zycki, Done \& Smith 1999) as the input continuum, which is primarily parametrized by the photon index of the continuum below the cut-off, and the electron temperature $\left(k T_{\mathrm{e}}\right)$. The key parameters for XILLVER_CP and RELXILLLP_CP are therefore essentially the same as for XILLVER and RELXILLLP, except that the high-energy cut-off parameter has been replaced with $k T_{\mathrm{e}}$. As before, we assume the same illuminating continuum parameters for both the RELXILLLP_CP and XILLVER_CP components, and we also continue with the approach taken in Model 3 and assume a common iron abundance for all AGN components.

\footnotetext{
${ }^{5}$ Table models for MYTORUs have also recently been computed for a realistic Compton-scattered illuminating continuum, but these use the сомРтт thermal Comptonization model (Titarchuk 1994) which has a different parametrization to NTHсомP, and these grids do not directly include the electron temperature as a free parameter.
} 


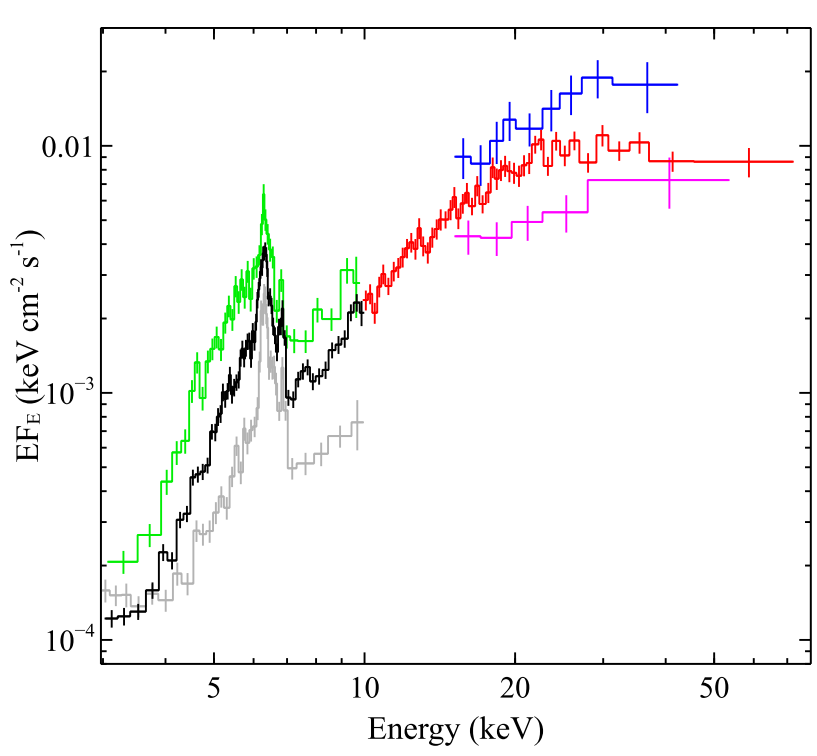

Figure 6. Multi-epoch spectra observed from IRAS 13197-1627. As in Fig. 3, the data have been unfolded through a model that is constant with energy, and we only show epochs S1 (high-flux data sets), S2 (low-flux data sets) and XN1 (medium-flux data sets), as epochs X1 and X2 are similar to epoch S1. For clarity, we only show the EPIC-pn data from XMM-Newton (black; below $10 \mathrm{keV}$ ), the FPMA data from NuSTAR (red; above $10 \mathrm{keV}$ ), and the data from the FI XIS detectors (S1: green, S2: grey) and the PIN detector (S1: blue, S2: magenta) from Suzaku. Epochs X1, X2 and S1 show lower levels of line-of-sight absorption than XN1, while epoch S2 has stronger absorption.

Model 4 also provides a similarly good fit to all the previous models considered (as before, see Table 2). The different curvature in the primary continuum allows in this case for lower values of $R_{\text {frac }}$, as the sharper curvature in the primary continuum allows this component to account for a bit more of the observed high-energy curvature by decreasing the electron temperature, a degeneracy that is naturally exacerbated by the strong absorption in the system. The formal constraint on the spin therefore weakens a little further in comparison to Model 3; here we find $a^{*}=0.72_{-0.53}^{+0.23}$. The rest of the parameters remain broadly similar to the results with Model 3.

Finally, although we do not present the full results to these fits as additional models here, we note that if we repeat the fit assuming the distant model components to have a solar abundance, but assuming a Comptonized continuum instead of a cut-off power law, we find practically identical results to those presented for Model 1. In this scenario, the precise form of the continuum does not have any significant effect. We also note that allowing the iron abundances for both the distant and the disc components to vary independently does not result in any significant statistical improvement over the common iron abundance scenario.

\subsection{Multi-epoch analysis}

In order to obtain the most robust constraints on the key inner disc parameters, in addition to our analysis of epoch XN1 we also undertake a multi-epoch analysis of all the observations included in Table 1. As with XN1, we only analyse the data above $3 \mathrm{keV}$. A comparison of some of the spectra from these observations is shown in Fig. 6. During the first three of these archival observations (X1, X2, S1), IRAS 13197-1627 was less absorbed than during epoch $\mathrm{XN1}$, and the spectra of these three data sets over their common $3-10 \mathrm{keV}$ bandpass were extremely similar. In contrast, during the last of the archival observations (S2), IRAS 13197-1627 was significantly more absorbed than XN1. Despite the changing absorption, the Suzaku PIN detections from S1 and S2 suggest that the intrinsic variations are relatively minor; the observed $20-40 \mathrm{keV}$ fluxes only vary by a factor of $\sim 2$.

We primarily focus on applying Model 4 (common iron abundance for all AGN model components, realistic Comptonized continuum) from Section 4.1 to all these observations simultaneously. All parameters that should not vary on observational time-scales, i.e. the black hole spin, disc inclination and iron abundance, are linked across all the data sets. In addition to these, we assume that the flux of the distant reflection is constant across all epochs, and also assume that the primary continuum parameters $\left(\Gamma, k T_{\mathrm{e}}\right)$, and the ionization states for both the accretion disc and the ionized absorption are constant across all epochs, given that the intrinsic flux variations between all the different epochs appear to be quite minor. Finally, given the strong similarity between the X1, X2 and S1 3-10 keV spectra, we also assume that the neutral absorption parameters are the same for these three epochs.

Within this framework, we test two possible explanations for the AGN variability that is present in addition to the absorption variability observed. First, we assume that the geometric structure of the inner accretion flow is static, and that all the variability is produced through intrinsic changes in the brightness of the corona. In modelling terms, we therefore keep $h$ constant across all epochs, and allow the normalization of the RELXILLLP_CP component to vary. Secondly, we assume that the intrinsic brightness of the corona is stable, and that the observed flux variations are produced through geometric changes that vary the degree of lightbending and thus the fraction of the primary emission that escapes directly to the observer, i.e. we allow $h$ to vary and keep the RELXILLLP_CP normalization constant. Lower source heights result in stronger lightbending, reducing the observed flux of the primary continuum (e.g. Miniutti \& Fabian 2004). Of course, both of these processes can occur simultaneously so the reality likely lies somewhere in between, but these are useful idealizations that reduce parameter degeneracies and show the two limiting scenarios. This is broadly similar to the approach taken in Walton et al. (2017).

The global fit obtained for the first scenario (intrinsic variations) is very good $\left(\chi^{2} / \mathrm{DoF}=1673 / 1545\right)$, and the parameter constraints are given in Table 3 . Many of the results from this analysis are broadly similar to those obtained with Model 4 considering epoch $\mathrm{XN1}$ alone. As expected, we see significant variations in the neutral absorption column comparing all the epochs. However, even during the most absorbed epoch (S2), the best-fitting column for the line-ofsight absorption is not Compton thick. This is further supported by the fact that there is reasonable evidence that the ionized absorption - which must arise closer to the black hole than the neutral absorber - is still visible in the data from this epoch; removing this component from the fit to $S 2$ degrades the fit by $\Delta \chi^{2}=13$ for two fewer free parameters.

For the black hole spin, we do indeed find that the formal parameter constraints are tighter than for XN1 alone; a comparison of the confidence contours obtained for XN1 and our multi-epoch analysis is shown in Fig. 7. In this case, the model prefers a high- $R_{\text {frac }}$, highspin scenario, with the spin constrained to $a^{*}>0.92$. However, as the spin decreases, the model quickly enters a region of parameter space in which the fit is not sensitive to this parameter, and a non-rotating black hole is again not excluded at high confidence. This is related to the same parameter degeneracy between $R_{\text {frac }}$ and $k T_{\mathrm{e}}$ discussed above (Section 4.1.4). At low spins, we find that the best-fitting electron temperature runs to $20 \mathrm{keV}$, the lower limit of 
Table 3. Results obtained for the free parameters in our multi-epoch lamppost reflection model fit.

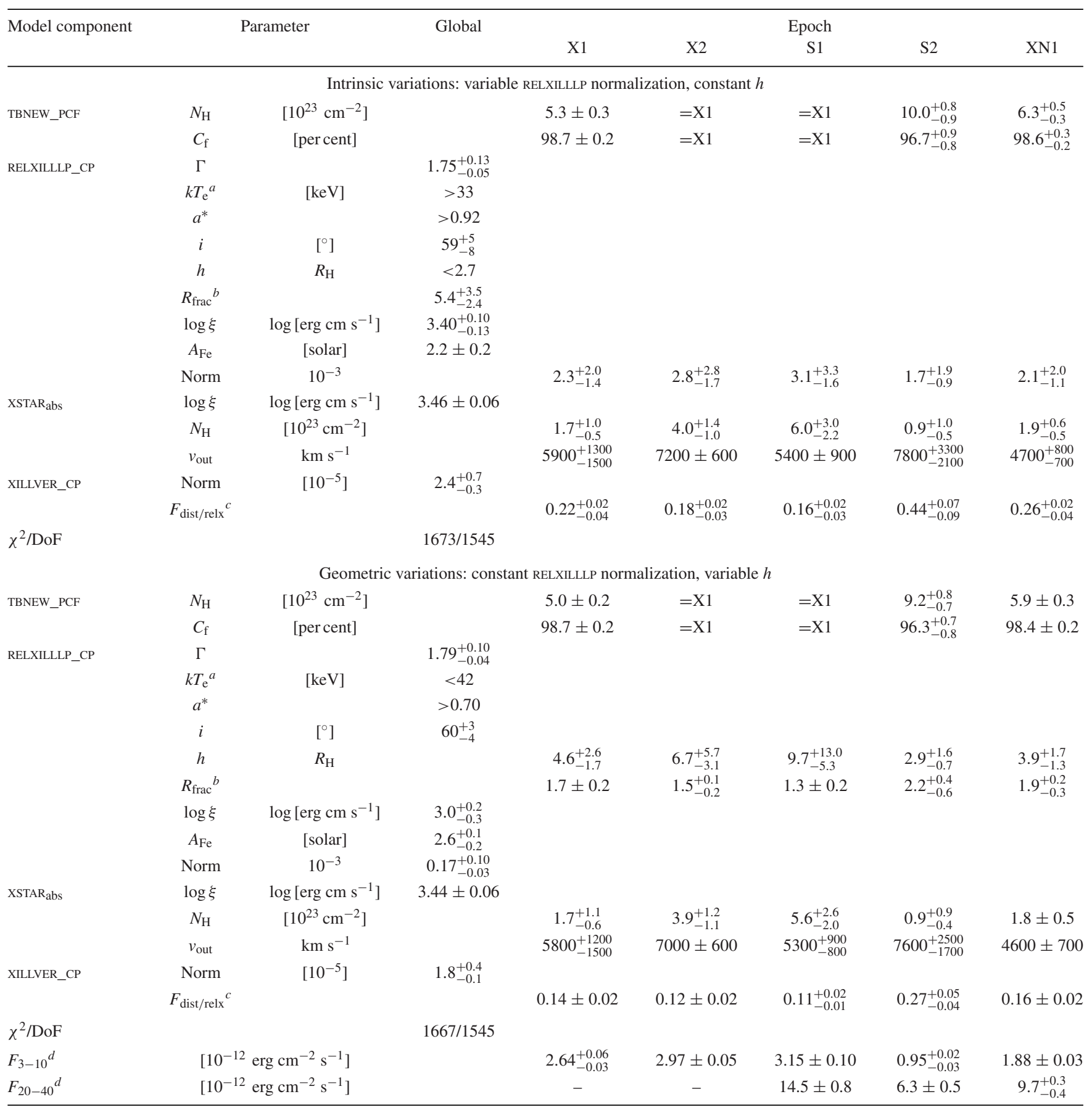

${ }^{a} k T_{\mathrm{e}}$ is limited by the bounds of the RELXILL_CP grid to $20 \leq k T_{\mathrm{e}} \leq 400 \mathrm{keV}$.

${ }^{b} R_{\text {frac }}$ is calculated self-consistently for a simple lamppost geometry from $a^{*}$ and $h$. Errors represent the range of values permitted by varying these parameters within their 90 per cent uncertainties.

${ }^{c}$ Ratio of the observed fluxes from the XILLVER_CP and the RELXILLLP_CP components in the $20-40 \mathrm{keV}$ band.

${ }^{d}$ Observed fluxes, not corrected for the line-of-sight absorption. These are consistent for both models.

the current RELXILLLP_CP grids. Such low coronal temperatures are highly unusual among the unobscured AGN population (see e.g. Fabian et al. 2015, though rare exceptions may be possible; Kara et al. 2017; Tortosa et al. 2017). We therefore re-compute the confidence contour for the spin holding $k T_{\mathrm{e}}$ fixed at $50 \mathrm{keV}$ (also shown in Fig. 7), which is both the best-fitting value for this scenario and also roughly the average value observed from the unobscured AGN compiled in Fabian et al. (2015). In this case, the formal 90 per cent statistical constraint is unchanged, but we see that low values for the black hole spin can be more confidently excluded. We stress that despite the degeneracy between $R_{\text {frac }}$ and $k T_{\mathrm{e}}$, the disc reflection contribution is strongly required by the data. Removing this from the fit (i.e. setting $R_{\text {frac }}=0$ ) degrades the fit by $\Delta \chi^{2}=102$ for four fewer free parameters. 


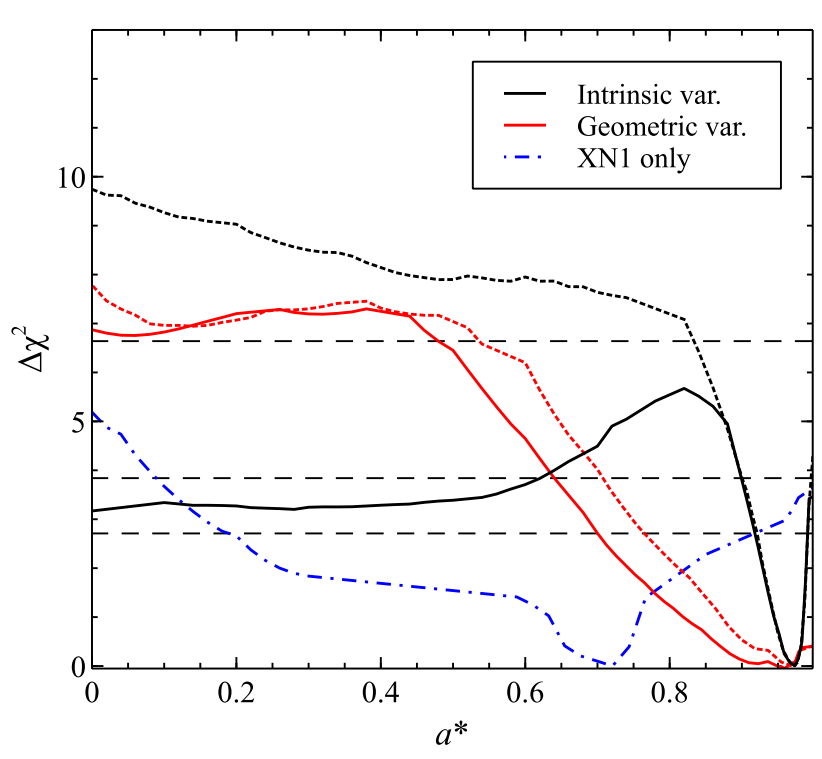

Figure 7. The $\Delta \chi^{2}$ confidence contours for the black hole spin obtained for our multi-epoch analysis using Model 4 . The black and red curves show the results assuming that the AGN variability is dominated by intrinsic and geometric changes in the corona, respectively, and the solid and dotted lines show the results for a free electron temperature and assuming $k T_{\mathrm{e}}=50 \mathrm{keV}$, respectively. As before, horizontal dashed lines represent the 90, 95 and 99 per cent confidence levels for a single parameter of interest. We also show the contour for epoch XN1 only from Fig. 4 for comparison (dash-dotted blue).

The second scenario (geometric variations) also provides an equally good fit $\left(\chi^{2} / \mathrm{DoF}=1667 / 1545\right)$. The parameter constraints are again given in Table 3 and, aside from the enforced differences, there are many similarities with the intrinsic variation scenario. In particular, the absorption variations are practically identical between the two scenarios. With this scenario, we find that the spin is constrained to be $a^{*}>0.70$ (see Fig. 7). This is not quite as tight as the intrinsic variation scenario, but the degeneracy between $R_{\text {frac }}$ and $k T_{\mathrm{e}}$ is not as severe in this case. This is because the bestfitting electron temperature is already unusually low $(\sim 23 \mathrm{keV})$, so the model cannot decrease $k T_{\mathrm{e}}$ to this level in order to fit lower reflection fractions, and thus lower spins (we stress though that while $k T_{\mathrm{e}}$ is already low, as we decrease the spin the model does not ever hit the lower limit of $20 \mathrm{keV}$ in the current version of RELXILLLP_CP, resulting in artificial increases in $\chi^{2}$ ). However, since the best-fitting electron temperature is unusually low, we again recompute the spin contour with $k T_{\mathrm{e}}=50 \mathrm{keV}$. This worsens the fit a little $\left(\chi^{2} / \mathrm{DoF}=1670 / 1546\right)$ and slightly contracts the formal spin constraint to $a^{*}>0.78$, but otherwise does not result in any other major changes to the confidence contour.

Finally, we also revisit the scenario in which the distant components are assumed to have a solar abundance with the multi-epoch data. Here, we continue to use the reflection models that assume realistic Comptonized spectra as the illuminating continuum, and also the broader approach taken in this section in terms of the parameters assumed to be constant and to vary between epochs, but return to decoupling the abundances of the disc components and the distant components, forcing the latter to have a solar abundance. As with our analysis of epoch XN1, we again find that the abundance of the disc components becomes highly super-solar $\left(A_{\mathrm{Fe}}>9.0\right)$. However, even if we allow both $h$ and the RELXILLLP_CP normalization to vary simultaneously, with the multi-epoch data the fit is notably worse than either of the cases in which all the model components are assumed to have a common iron abundance: $\chi^{2} / \mathrm{DoF}$ $=1719 / 1545$, i.e. $\Delta \chi^{2}>45$ worse despite having more degrees of freedom. We can therefore confidently exclude this possibility thanks to the multi-epoch data set, and so we do not present the results for this model in any further detail.

\section{DISCUSSION}

We have presented a broad-band X-ray spectral analysis of a new coordinated XMM-Newton+NuSTAR observation of the type 1.8 Seyfert galaxy IRAS 13197-1627, along with a multi-epoch $\mathrm{X}$-ray analysis further incorporating archival observations taken with XMM-Newton and Suzaku. The broad-band X-ray spectrum exhibited by this source is highly complex, with strong contributions from relativistic reflection from the inner accretion disc, absorption and further reprocessing by more distant material, and ionized absorption from an outflow all combining to sculpt the observed spectral form, similar to the well-studied AGN in NGC 1365 (Risaliti et al. 2013; Walton et al. 2014; Rivers et al. 2015). By combining the high-S/N broad-band data provided by XMM-Newton and NuSTAR with the multi-epoch archival data obtained with XMM-Newton and Suzaku, we are able to disentangle the relative contributions from all these various components for a variety of different scenarios, allowing us to place constraints on the parameters of the innermost accretion flow, and in turn the spin of the black hole, despite the heavy absorption present in this system.

\subsection{Metallicity and the inner disc}

Based on our analysis of epoch $\mathrm{XN1}$, the key inner disc parameters (e.g. black hole spin, disc inclination) appear to be largely independent of the precise treatment of the distant reflection (slab versus torus geometry) and are only moderately sensitive to the form of the primary continuum (power law with an exponential cut-off versus realistic Comptonized continuum). However, the results are strongly dependent on the treatment of the iron abundance for the neutral absorption (and by extension the distant reflection, which we assume to be associated with the same medium).

We test two different scenarios for the iron abundance, focusing initially on epoch $\mathrm{XN} 1$ as the highest $\mathrm{S} / \mathrm{N}$ broad-band data set. First, we assume that the neutral absorption/distant reprocessor has a solar iron abundance - as is often the case in the literature - but allow the abundance for the disc components to vary, and secondly we assume that all the model components have a common iron abundance, which is free to vary. In the former scenario, we find that the contribution from the disc reflection needs to be very strong $\left(R_{\text {frac }} \gtrsim 3\right)$ in order to model the strong drop in the spectrum at $\sim 7 \mathrm{keV}$ and the curvature of the continuum above $10 \mathrm{keV}$ (see Fig. 3). This requires the X-ray source to be very close to a rapidly rotating black hole, such that strong gravitational lightbending can sufficiently enhance the disc reflection relative to the observed primary continuum emission (e.g. Miniutti \& Fabian 2004). Combining the constraints from the slab and torus models for the distant reflection (Models 1 and 2, respectively), we find the black hole spin to be $0.89 \leq a^{*} \leq 0.98$ in this scenario. However, in this scenario, a large discrepancy between the iron abundance assumed for the distant material and that inferred for the disc is seen, with the disc required to be strongly super-solar $\left(A_{\mathrm{Fe}}>7.1\right)$. Assuming instead that all model components are chemically homogeneous, we find a moderately super-solar iron abundance $\left(A_{\mathrm{Fe}} \sim 2.3\right)$ and that the requirement for strong reflection and gravitational lightbending 
is much less severe (although such solutions are still permitted). As such, the constraints on the spin are subsequently much looser; considering epoch $\mathrm{XN1}$ only, we could only constrain the spin to $0.19 \leq a^{*} \leq 0.98$. Neither of these scenarios is strongly preferred over the other in a statistical sense for the broad-band XN1 data, although the common iron abundance scenario does result in a minor improvement to the fit (see Table 2).

The scenario in which the iron abundance is common to all AGN model components is clearly the more intuitive of these two possibilities, and would likely be the preferred scenario in the situation where only the broad-band data from epoch XN1 were available. In addition, the higher inclination inferred in the chemically homogeneous scenario $\left(\sim 60^{\circ}\right)$ is probably more in line with expectation for a system with such high levels of absorption. However, the scenario in which the distant components have a solar abundance cannot statistically be ruled out with these data alone. Furthermore, although such measurements are notoriously challenging, the metallicity indicator based on the $\mathrm{C}_{\text {III }}, \mathrm{C}_{\text {IV }}$ and $\mathrm{He}_{\text {II }}$ lines presented by Dors et al. (2014) suggests that the narrow-line region (NLR) in IRAS 13197-1627 has a solar metallicity. A solar iron abundance could therefore also be expected for the distant model components in our work, which would still appear to be in some tension with the super-solar abundance inferred in the chemically homogeneous scenario. There may therefore be some justification for preferring the chemically inhomogeneous scenario if both are statistically permissible. This would in turn require a mechanism by which the inner accretion disc could have, or at least appear to have, a significantly different abundance than the neutral absorber/distant reprocessor.

Two potentially interesting possibilities for such a mechanism are discussed in Reynolds et al. (2012). The first is that the atomic iron in the distant absorber/reprocessor could be depleted into dust grains. Should these grains be sufficiently large, self-shielding could reduce the strength of the atomic iron signatures relative to the contribution of the distant backscattered continuum (i.e. the distant Compton hump), which would give the appearance of a reduced iron abundance for the distant model components. Secondly, Reynolds et al. (2012) also discuss the possibility that the photospheric iron abundance of the inner disc could appear to be enhanced by radiative levitation of iron atoms to the disc surface. In this scenario, the background radiation field within the innermost regions of an AGN accretion disc could produce a net upward force on moderately ionized iron, causing the iron to diffuse upwards. Some evidence for this process may have been observed recently from the Galactic black hole X-ray binary GRS $1915+105$, where the iron abundance appears to change across the phase of the $50 \mathrm{~s}$ limit cycle oscillations observed from this source (Zoghbi et al. 2016).

These possibilities are not without their problems. With regard to potential dust depletion of iron in the distant absorber/reprocessor, while the NLR should reside beyond the dust sublimation radius (Netzer 2015), we note that there is evidence that the majority of the narrow core to the iron emission in AGN may arise from regions interior to this (Gandhi, Hönig \& Kishimoto 2015). Furthermore, Reynolds et al. (2012) note that radiative levitation of iron may only be relevant for high accretion rates (close to the Eddington limit) on to lower mass AGN, where disc temperatures are higher. The mass of IRAS 13197-1627 is not well known, but Vasudevan et al. (2010) estimate the mass and bolometric luminosity to be $\log \left(M_{\mathrm{BH}} / \mathrm{M}_{\odot}\right) \sim 7.81$ and $L_{\mathrm{bol}} \sim 5-8 \times 10^{44} \mathrm{erg} \mathrm{s}^{-1}$, respectively, combining X-ray and infrared information. These values would correspond to $L_{\mathrm{bol}} / L_{\mathrm{Edd}} \sim 0.05-0.1$. While this is still fairly uncertain, the accretion rate is likely too low for radiative levitation to play an important role in this case. However, although these processes may struggle individually to produce a metallicity gradient large enough to match that inferred here, they could potentially act in combination. Furthermore, there may be other mechanisms beyond those considered here that can produce strong/additional metallicity gradients (actual or apparent), so it would also be difficult to conclusively rule out the chemically inhomogeneous scenario through physical arguments based on epoch XN1 alone.

However, we find that when considering the multi-epoch data set - additionally including archival observations from XMM-Newton and Suzaku (epochs X1, X2, S1, S2) which show both varying levels of absorption and intrinsic variability from the central AGN - we can distinguish between these two scenarios in a statistical sense, with the chemically homogeneous scenario clearly preferred. This clearly demonstrates the importance of combining high- $\mathrm{S} / \mathrm{N}$ broadband observations with a multi-epoch approach to disentangle the various emission and absorption components for these complex AGN, particularly when the level of absorption is variable, and the typical column is as high as it is for IRAS 13197-1627.

Within the chemically homogeneous scenario, we test two different possible explanations for the AGN variability observed. First, we assume that this is dominated by intrinsic brightness variations in the corona, and secondly we assume that it is dominated by variations in the geometry of the corona that change the degree of lightbending, resulting in variations in the observed flux. Both of these limiting scenarios fit the multi-epoch data similarly well, and both improve the formal spin constraint in comparison to the fits to epoch $\mathrm{XN1}$ alone. When the variations are assumed to be intrinsic, we find $a^{*}>0.92$, and when they are assumed to be geometric we find $a^{*}>0.70$. In these fits, we often find that the electron temperature moves into an unusually low area of parameter space $\left(k T_{\mathrm{e}} \sim 20 \mathrm{keV}\right)$. If we make sensible assumptions regarding this temperature based on the results seen in unobscured systems (e.g. Fabian et al. 2015, and references therein) and assume $k T_{\mathrm{e}}=50 \mathrm{keV}$, in the intrinsic variation scenario we find that slowly rotating black holes can be more confidently excluded (but the formal 90 per cent spin constraint is unchanged), and in the geometric variation scenario the spin constraint improves to $a^{*}>0.78$. However, we stress that a slowly rotating black hole is not very strongly excluded for any of these scenarios, so the spin constraint presented here would certainly benefit from confirmation with further broad-band observations, ideally probing lower levels of obscuration than seen during epoch XN1. To be conservative, we take $a^{*}>0.7$ as our final spin constraint.

\subsection{Variations in the neutral absorber}

From our multi-epoch analysis, we find evidence that the line-ofsight column of the neutral absorber varies from epoch to epoch, ranging over $N_{\mathrm{H}} \sim 5-10 \times 10^{23} \mathrm{~cm}^{-2}$, suggesting that the absorbing medium has a clumpy structure. A hint of this was previously claimed by Risaliti, Elvis \& Nicastro (2002) comparing archival observations by BeppoSAX and ASCA, but the subsequent analysis by Miniutti et al. (2007) found the column density between these observations and the first XMM-Newton epoch (X1) to be consistent. Although we have made simplifying assumptions in our analysis, e.g. forcing the photon index and the height of the X-ray reflector to be constant across all epochs, the changes in $N_{\mathrm{H}}$ observed here are too strong for these assumptions to have any major influence on this conclusion. Assuming that this absorption is related to the torus structure invoked in AGN unification schemes, this could add further observational evidence to the suggestion that this torus is clumpy, rather than a uniform structure (e.g. Nikutta, 
Elitzur \& Lacy 2009; Markowitz, Krumpe \& Nikutta 2014). However, it is also possible that the absorbing clouds are located instead in the broad-line region, as appears to be the case in NGC 1365 (e.g. Risaliti et al. 2009). Unfortunately, the observations presented here are separated by $\sim$ years, so a meaningful test of the location of the absorbing clouds is not currently possible.

If the neutral absorber is associated with the distant reflector, as we have generally assumed here, then the apparent stability of this emission over the full set of observations could suggest that this material is located at distances $\gtrsim 10$ light-years, or equivalently $\gtrsim 10^{6} R_{\mathrm{G}}$ for a mass of $\log \left(M_{\mathrm{BH}} / \mathrm{M}_{\odot}\right) \sim 7.81$, given the full temporal separation of the observations considered. This would likely place the material beyond the broad-line region (Netzer 2015). However, we note again that the variations in the intrinsic flux between epochs are fairly minor (less than a factor of 2, after accounting for the scattering losses; see the variations in the RELXILLLP normalization in Table 3), so it may be that the average flux on long time-scales is relatively constant. If this is the case, then we may not expect to see strong variations in the distant reflector, even if this material is located at radii smaller than $10^{6} R_{\mathrm{G}}$. All of this could be tested in the future with higher cadence broad-band observations, both to study the intrinsic flux variations and the time-scales on which the absorption varies.

In contrast to the column density, the covering fraction obtained for the neutral absorber is persistently high ( $\gtrsim 97$ per cent). Covering fractions this high are likely indicative that, although the model is formally partially covering, along our line of sight the absorber is actually fully covering in a geometric sense, and the non-unity covering fraction is accounting for the small fraction of the intrinsic continuum emission that gets scattered around the absorber and back into our line of sight. Indeed, X-ray obscured AGN often display scattered fractions at the $\sim$ few per cent level (e.g. Ichikawa et al. 2012), similar to that inferred here.

\subsection{An ionized outflow}

In addition to the relativistic reflection and strong neutral absorption that strongly influence the broad-band spectrum observed, we also find robust evidence that IRAS 13197-1627 exhibits blueshifted absorption from ionized iron, with absorption lines from both Fe XXV and Fe XXVI observed, confirming the tentative indication reported in previous works (Dadina \& Cappi 2004; Miniutti et al. 2007). Evidence for this absorption is seen at all epochs analysed in this work, but its properties appear to be variable, with the line-ofsight column density and outflow velocity ranging from $N_{\mathrm{H}} \sim 2-$ $6 \times 10^{23} \mathrm{~cm}^{-2}$ (assuming that the system is chemically homogeneous) and from $v_{\text {out }} \sim 5000-8000 \mathrm{~km} \mathrm{~s}^{-1}$, respectively. These velocities are not dissimilar to the ionized outflow seen in NGC 1365 (Risaliti et al. 2005), but are not as extreme as the 'ultra-fast' outflows now robustly confirmed with broad-band spectroscopy in a few AGN (e.g. Nardini et al. 2015; Lobban et al. 2016; Parker et al. 2017).

Throughout this work, we have assumed that the ionized absorber is associated with the accretion disc, and therefore physically interior to the neutral absorber. Given the high degree of ionization, and the fairly rapid outflow velocity, it is natural to assume that this absorption would occur closer to the central ionizing source. However, it is worth noting that since we use multiplicative absorption models, and both apply to the central emission from the source, the results obtained for the inner disc do not depend on this assumption for any of the models considered. The only issue this relates to is the iron abundance assumed for the ionized absorption in the chemically inhomogeneous models (i.e. whether this should be linked to the disc components or the distant components). However, since the only features the ionized absorption contributes to the observed spectrum are the iron absorption lines, the only result that will be influenced by this assumption is the column density inferred for this component (which is strongly degenerate with the iron abundance as a consequence). In the (preferred) chemically homogeneous models, the absorbers are fully commutative in a functional sense, since the iron abundance is the same for all model components.

Although the values obtained for the column density of the ionized absorption do depend heavily on our treatment of the iron abundance, this only serves to set the overall scale for the column density results, and cannot explain the variations in the column seen between epochs. This variability suggests that there is also some level of inhomogeneity in the ionized wind, in addition to the neutral absorption, although the variations in the ionized absorption do not obviously appear to correlate (or anti-correlate) with those in the neutral absorber. Such inhomogeneities are likely also expected for disc winds; magnetohydrodynamic simulations of disc winds show them to be clumpy, time-variable structures (e.g. Proga $\&$ Kallman 2004). However, the fact that this ionized absorption is seen at all epochs likely suggests that along our line of sight, these inhomogeneities may not be too severe.

\section{CONCLUSIONS}

The X-ray spectrum of IRAS 13197-1627 is highly complex, exhibiting contributions from relativistic reflection from the inner accretion disc, absorption and further reprocessing by more distant material, and absorption from an ionized outflow. By utilizing the high-sensitivity, broad-band coverage provided by $X M M-$ Newton $+N u S T A R$, as well as a multi-epoch approach incorporating the archival observations performed by XMM-Newton and Suzaku, we perform detailed spectral analysis with an emphasis on separating out the reflection from the innermost accretion disc, which previous works have suggested may dominate the AGN emission. Using the latest reflection and absorption models and focusing first on the broad-band XMM-Newton+NuSTAR epoch, we find that the results for the inner disc do not strongly depend on the geometry assumed for the distant reprocessor, or the precise form of the illuminating X-ray continuum. However, these results do depend on the treatment of the iron abundance of the distant absorber/reprocessor. If this is assumed to have a solar abundance, as may be suggested by independent metallicity estimates for the NLR, a high-spin, reflection-dominated scenario is strongly required $\left(0.89 \leq a^{*} \leq\right.$ 0.98 ), but the results also require a highly super-solar abundance for the disc $\left(A_{\mathrm{Fe}}>7.1\right)$. If we instead assume that the system is chemically homogeneous and link the iron abundance between all the AGN components, we find a moderately super-solar abundance $\left(A_{\mathrm{Fe}}=2.3_{-0.1}^{+0.2}\right)$ and the constraint on the spin is significantly weaker $\left(0.19 \leq a^{*} \leq 0.98\right)$.

Both of these scenarios fit the XMM-Newton+NuSTAR data similarly well. However, when we incorporate the data from archival $X M M-N e w t o n$ and Suzaku observations, and fit these data sets simultaneously with the $X M M-N e w t o n+N u S T A R$ epoch, we find that the chemically homogeneous scenario is preferred in a statistical sense thanks to the absorption variability observed. This demonstrates the importance of combining both broad-band and multi-epoch spectroscopy for AGN with sources as complex as IRAS 13197-1627. Including these data also improves the formal spin constraint to $a^{*}>0.7$ for this scenario, so a rapidly rotating 
black hole is preferred. However, a slowly rotating black hole is still not strongly excluded, so this constraint should be confirmed with additional broad-band observations.

In addition to the results for the inner disc, through our multiepoch analysis we also find that both the neutral and ionized absorbers vary from epoch to epoch. This suggests that both the neutral absorber, either the torus or broad-line region clouds, and the ionized absorber, an accretion disc wind, have an inhomogeneous, clumpy structure (at least to some extent). Higher cadence broad-band monitoring in the future should be able to constrain the location of the variable neutral absorber and determine whether this is associated with the torus or the broad-line region.

\section{ACKNOWLEDGEMENTS}

The authors would like to thank the reviewer for their helpful feedback, which helped to improve the final version of the manuscript. DJW acknowledges support from an STFC Ernest Rutherford Fellowship, and ACF acknowledges support from ERC Advanced Grant 340442. GM thanks the European Union Seventh Framework Program (FP7/2007-2013) for funding under grant 312789 (StrongGravity). This research has made use of data obtained with NuSTAR, a project led by Caltech, funded by NASA and managed by NASA/JPL, and has utilized the NUSTARDAS software package, jointly developed by the ASDC (Italy) and Caltech (USA). This research has also made use of data obtained with XMM-Newton, an ESA science mission with instruments and contributions directly funded by ESA Member States, and with Suzaku, a collaborative mission between the space agencies of Japan (JAXA) and the USA (NASA).

\section{REFERENCES}

Anders E., Grevesse N., 1989, Geochim. Cosmochim. Acta, 53, 197

Arnaud K. A., 1996, in Jacoby G. H., Barnes J., eds, ASP Conf. Ser. Vol. 101, Astronomical Data Analysis Software and Systems V. Astron. Soc. Pac., San Francisco, p. 17

Boldt E., 1987, in Hewitt A., Burbidge G., Fang L. Z., eds, Proc. IAU Symp. 124, Observational Cosmology. Reidel, Dordrecht, p. 611

Bonson K., Gallo L. C., 2016, MNRAS, 458, 1927

Brenneman L. W. et al., 2011, ApJ, 736, 103

Brightman M., Nandra K., 2011, MNRAS, 413, 1206

Brightman M. et al., 2015, ApJ, 805, 41

Dadina M., Cappi M., 2004, A\&A, 413, 921

Dauser T., Wilms J., Reynolds C. S., Brenneman L. W., 2010, MNRAS, 409,1534

Dauser T., García J., Parker M. L., Fabian A. C., Wilms J., 2014, MNRAS, 444, L100

Dauser T., García J., Walton D. J., Eikmann W., Kallman T., McClintock J., Wilms J., 2016, A\&A, 590, A76

Dors O. L., Cardaci M. V., Hägele G. F., Krabbe Â. C., 2014, MNRAS, 443, 1291

Dubois Y., Volonteri M., Silk J., 2014, MNRAS, 440, 1590

Fabian A. C., Rees M. J., Stella L., White N. E., 1989, MNRAS, 238, 729

Fabian A. C. et al., 2009, Nature, 459, 540

Fabian A. C. et al., 2013, MNRAS, 429, 2917

Fabian A. C., Lohfink A., Kara E., Parker M. L., Vasudevan R., Reynolds C. S., 2015, MNRAS, 451, 4375

Fürst F. et al., 2016, ApJ, 819, 150

Gandhi P., Hönig S. F., Kishimoto M., 2015, ApJ, 812, 113

García J., Kallman T. R., 2010, ApJ, 718, 695

García J. et al., 2014, ApJ, 782, 76

García J. A., Dauser T., Steiner J. F., McClintock J. E., Keck M. L., Wilms J., 2015, ApJ, 808, L37
Grevesse N., Sauval A. J., 1998, Space Sci. Rev., 85, 161

Haardt F., Maraschi L., 1991, ApJ, 380, L51

Harrison F. A. et al., 2013, ApJ, 770, 103

Ichikawa K., Ueda Y., Terashima Y., Oyabu S., Gandhi P., Matsuta K., Nakagawa T., 2012, ApJ, 754, 45

Jansen F. et al., 2001, A\&A, 365, L1

Kalberla P. M. W., Burton W. B., Hartmann D., Arnal E. M., Bajaja E., Morras R., Pöppel W. G. L., 2005, A\&A, 440, 775

Kallman T., Bautista M., 2001, ApJS, 133, 221

Kara E. et al., 2017, MNRAS, 468, 3489

Koyama K. et al., 2007, PASJ, 59, 23

Lansbury G. B. et al., 2017, ApJ, 846, 20

Laor A., 1991, ApJ, 376, 90

Lobban A. P., Pounds K., Vaughan S., Reeves J. N., 2016, ApJ, 831, 201

Madsen K. K. et al., 2015, ApJS, 220, 8

Mantovani G., Nandra K., Ponti G., 2016, MNRAS, 458, 4198

Markowitz A. G., Krumpe M., Nikutta R., 2014, MNRAS, 439, 1403

Miller L., Turner T. J., Reeves J. N., 2008, A\&A, 483, 437

Miller L., Turner T. J., Reeves J. N., 2009, MNRAS, 399, L69

Miniutti G., Fabian A. C., 2004, MNRAS, 349, 1435

Miniutti G., Ponti G., Dadina M., Cappi M., Malaguti G., 2007, MNRAS, 375,227

Murphy K. D., Yaqoob T., 2009, MNRAS, 397, 1549

Nardini E., Fabian A. C., Reis R. C., Walton D. J., 2011, MNRAS, 410, 1251

Nardini E. et al., 2015, Science, 347, 860

Netzer H., 2015, ARA\&A, 53, 365

Nikutta R., Elitzur M., Lacy M., 2009, ApJ, 707, 1550

Parker M. L. et al., 2014, MNRAS, 443, 1723

Parker M. L. et al., 2017, Nature, 543, 83

Proga D., Kallman T. R., 2004, ApJ, 616, 688

Reis R. C., Reynolds M. T., Miller J. M., Walton D. J., 2014, Nature, 507, 207

Reynolds C. S., 2014, Space Sci. Rev., 183, 277

Reynolds C. S., Brenneman L. W., Lohfink A. M., Trippe M. L., Miller J. M., Fabian A. C., Nowak M. A., 2012, ApJ, 755, 88

Reynolds M. T., Walton D. J., Miller J. M., Reis R. C., 2014, ApJ, 792, L19

Risaliti G., Elvis M., Nicastro F., 2002, ApJ, 571, 234

Risaliti G., Bianchi S., Matt G., Baldi A., Elvis M., Fabbiano G., Zezas A., 2005, ApJ, 630, L129

Risaliti G. et al., 2009, MNRAS, 393, L1

Risaliti G. et al., 2013, Nature, 494, 449

Rivers E., Markowitz A., Rothschild R., 2013, ApJ, 772, 114

Rivers E. et al., 2015, ApJ, 804, 107

Sesana A., Barausse E., Dotti M., Rossi E. M., 2014, ApJ, 794, 104

Sim S. A., Proga D., Miller L., Long K. S., Turner T. J., 2010, MNRAS, 408, 1396

Strüder L. et al., 2001, A\&A, 365, L18

Takahashi T. et al., 2007, PASJ, 59, 35

Tanaka Y. et al., 1995, Nature, 375, 659

Tatum M. M., Turner T. J., Miller L., Reeves J. N., 2013, ApJ, 762, 80

Titarchuk L., 1994, ApJ, 434, 570

Tortosa A. et al., 2017, MNRAS, 466, 4193

Turner M. J. L. et al., 2001, A\&A, 365, L27

Vasudevan R. V., Fabian A. C., Gandhi P., Winter L. M., Mushotzky R. F., 2010, MNRAS, 402, 1081

Vasudevan R. V., Fabian A. C., Reynolds C. S., Aird J., Dauser T., Gallo L. C., 2016, MNRAS, 458, 2012

Verner D. A., Ferland G. J., Korista K. T., Yakovlev D. G., 1996, ApJ, 465 , 487

Volonteri M., Sikora M., Lasota J.-P., Merloni A., 2013, ApJ, 775, 94

Walton D. J., Reis R. C., Fabian A. C., 2010, MNRAS, 408, 601

Walton D. J., Nardini E., Fabian A. C., Gallo L. C., Reis R. C., 2013, MNRAS, 428, 2901

Walton D. J. et al., 2014, ApJ, 788, 76

Walton D. J., Reynolds M. T., Miller J. M., Reis R. C., Stern D., Harrison F. A., 2015, ApJ, 805, 161

Walton D. J. et al., 2016, ApJ, 826, 87 
Walton D. J. et al., 2017, ApJ, 839, 110

Wilkins D. R., Fabian A. C., 2012, MNRAS, 424, 1284

Wilms J., Allen A., McCray R., 2000, ApJ, 542, 914

Xu Y., Balokovic M., Walton D. J., Harrison F. A., Garcia J. A., Koss M. J., 2017, ApJ, 837, 21

Zdziarski A. A., Johnson W. N., Magdziarz P., 1996, MNRAS, 283, 193
Zdziarski A. A., Lubiński P., Gilfanov M., Revnivtsev M., 2003, MNRAS, 342,355

Zoghbi A. et al., 2016, ApJ, 833, 165

Zycki P. T., Done C., Smith D. A., 1999, MNRAS, 309, 561

This paper has been typeset from a $\mathrm{T}_{\mathrm{E}} \mathrm{X} / \mathrm{L} \mathrm{T} \mathrm{E} \mathrm{X}$ file prepared by the author. 